\title{
Parameter estimation of Singh Maddala distribution by moments
}

\author{
Mirza Naveed Shahzad ${ }^{1 *}$, Zahid Asghar ${ }^{2}$ \\ ${ }^{1}$ Department of Statistics, University of Gujrat, Pakistan \\ ${ }^{2}$ Subject Specialist Econometrics, NIBAF, State Bank of Pakistan, Islamabad, Pakistan \\ *Corresponding author E-mail: nvd.shzd@uog.edu.pk
}

\begin{abstract}
Singh Maddala distribution is a flexible distribution and mostly used for modeling the income, wage, expenditure and wealth distribution of a country. Its accurate parameter estimation is required due to its worth use. The L-moments and TL-moments of the Singh Maddala distribution have derived in close form for the parameter estimation. These moments are used to estimate the scale parameter which is related to the inequality of the income distribution. This study shows that L-moments and TL-moments are better alternatives against conventional moments and estimators of these moments equally applicable for both small and large sample size. Through Monte Carlo simulation study, we found TL-moments estimators provide least bias, root mean square error, coefficient of variation, skewness and kurtosis.
\end{abstract}

Keywords: L-moments, TL-moments, conventional moments, parameters estimation, Singh Maddala distribution, simulation study.

\section{Introduction}

Singh Maddala Distribution (SMD) provides the overall better fit on the full range of income distribution data than Pareto, Lognormal and Gamma distribution ([18], [5]). Modeling the distribution of the income of a country is used to assess the living standards and income equality in the population. Mostly Generalized Beta distribution and its different types are used to model the income distribution. These distributions have been shown to give a better fit at the univariate level ([11]). The SMD also belongs to the family of Beta distributions.

L-moments and TL-moments provide efficient estimators for parameter estimation. As after the [9] L-moments are extensively used because by this method one can identify the best fitted distribution and estimate their parameters more accurately than conventional moments. TL-moments presented by [6] as the extension of L-moments, these moments are more robustness and can be calculated even if the mean of the distribution does not exist. Many statistical distributions are analyzed by L-moments. L-moments of Generalized Rayleigh distribution was derived by [12], LMoments and TL-Moments of Exponential distribution, of the Generalized Lambda Distribution, Generalized Pareto Distribution, Generalized Logistic Distribution, Dagum distribution, studied by [1], [3], [2], [13], [14] respectively. [9] Analyzed ten basic distributions in the initial research of L-moment. They all conclude that the L and TL moments performance was found to be better than other moments.

We derive the first four moments for L-moments and TL-moments of the SMD and using these we also derive the coefficient of variation (CV), coefficient of skewness (CS) and coefficient of kurtosis (CK) estimators. To date, to our knowledge there is no one who derived these moments for the SMD and then performed their evaluation. Taking the L and TL-estimators, we estimate the scale parameter of the SMD and compare it with central moments on the basis of biasness and Root Mean Square Error (RMSEs) to suggest the efficient estimator. We also compute the CV, CS and CK using the method of moment, L-moment and TL-moment estimators and compare them with the help of RMSEs. All comparisons are based on Monte Carlo simulation study in which we use different sample sizes and different parametric values for the SMD. This study will provide useful insight for modeling the income data taking the L-moments and TLmoments of the SMD.

In section 2 and 3 we introduce the theoretical and sample L-moments and TL-moments and their coefficient ratios for the SMD distribution. In section 4 we discuss the parameters, probability distribution, distribution function and conventional moments of the SMD. We derive the L and TL moments and the CV, CS and CK are also presented in section 5. Section 6 contains, a simulation based numerical study to calculate the estimates from the derived estimators and then compare the three considered method of estimation and section 7 concludes the paper. 


\section{Material and methods}

\subsection{Method of L-moments}

L-Moments introduced by [9] and derived these moments for well-known distributions and proved that L-moments have many theoretical advantages over ordinary moments. L-moments are computed from linear combinations of the ordered data values and can be defined for any random variable whose mean exists. So order statistics and quantile function are used to compute the L-moments. This method is used as a summary statistics such as measures of location, dispersion, skewness and kurtosis for probability distributions and estimation of parameters and quantiles of probability distributions and hypothesis testing of probability distributions. L-moments also provide better identification of the parent distribution which generated a particular data sample than the conventional moments. Furthermore, L-moments are less sensitive in the case of outlier in data ([16]).

Probability weighted moments (PWM) derived by [7] and it also express the relation to parameters of several distributions whose inverse forms are explicitly defined. And the L-moments are linear combinations of the PWM according to the [9]. The $r^{\text {th }}$ L-moments is defined as,

$$
\lambda_{r}=\frac{1}{r} \sum_{k=0}^{r-1}(-1)^{k} C_{k}^{r-1} E\left(X_{r-k: r}\right)
$$

Where $r$ is the $r^{\text {th }}$ L-moment of the distribution and the last term is the expected value of $X_{r-k: r}$ where (r-k) ${ }^{t h}$ smallest observation in a sample of size $n$. The first four linear moments of a random variable are as follows using the (1)

$$
\begin{aligned}
& \lambda_{1}=E(X)=\int_{0}^{1} x(F) d F \\
& \lambda_{2}=\frac{1}{2} E\left(X_{2: 2}-X_{1: 2}\right)=\int_{0}^{1} x(F)(2 F-1) d F \\
& \lambda_{3}=\frac{1}{3} E\left(X_{3: 3}-2 X_{2: 3}+X_{1: 3}\right)=\int_{0}^{1} x(F)\left(6 F^{2}-6 F-1\right) d F \\
& \lambda_{4}=\frac{1}{4} E\left(X_{4: 4}-3 X_{3: 4}+3 X_{2: 4}-X_{1: 4}\right)=\int_{0}^{1} x(F)\left(20 F^{3}-30 F^{2}+12 F-1\right) d F
\end{aligned}
$$

The $\lambda_{1} \& \lambda_{2}$ are the measures of location and dispersion respectively. The L-moment ratios computed from the Lmoments coefficients are defined as $\tau_{r}=\lambda_{r} / \lambda_{2}$, where $r \geq 3 . \tau_{3}=\lambda_{3} / \lambda_{2}$ and $\tau_{4}=\lambda_{4} / \lambda_{2}$ are the measure of skewness and measure of kurtosis respectively.

To calculate the sample L-moments we will assume that ' $\mathrm{X}$ ' as a random sample and ordered the sample value. Unbiased sample estimators of the PWMs are defined by the [8]. The first four sample estimators of the PWMs are as follow:

$$
\begin{aligned}
& b_{0}=\bar{X} \\
& b_{1}=\frac{1}{n(n-1)} \sum_{j=2}^{n}(j-1) X_{(j)} \\
& b_{2}=\frac{1}{n(n-1)(n-2)} \sum_{j=3}^{n}(j-1)(j-2) X_{(j)} \\
& b_{3}=\frac{1}{n(n-1)(n-2)(n-3)} \sum_{j=4}^{n}(j-1)(j-2)(j-3) X_{(j)}
\end{aligned}
$$

Sample L-moments are similarly to the conventional sample L-moments in properties. This relationship can be used to estimate the parameters of the distribution. Using the above PWMs sample estimators we can find the sample Lmoments $\left(l_{1}, l_{2}, l_{3}, l_{4}\right)$ as $l_{1}=b_{0}, l_{2}=2 b_{1}-b_{0}, l_{3}=6 b_{2}-6 b_{1}+b_{0}$ and $l_{4}=20 b_{3}-30 b_{2}+12 b_{1}-b_{0}$. Sample estimates for L-moments ratios can be computed as $t_{r}=l_{r} / l_{2}$, where $r \geq 3$.

\subsection{Method of TL-moments}

TL-moments introduced by [6] are more robust than L-moments. L-moments have the assumption of mean existence but the TL-moments can be estimated even when mean does not exist, [2] derived the TL-moments for Cauchy distribution. In the TL-moments a predetermined percentage of the outlier values are trimmed by assigning zero weight 
before estimating the moments. These moments are also used for obtaining the best fit distribution and to estimate the parameters for probability distributions. The $r$ th TL-moments as follows:

$$
\lambda_{r}^{(1)}=\frac{1}{r} \sum_{k=0}^{r-1}(-1)^{k} C_{k}^{r-1} E\left(X_{r+t+k: r+2 t}\right)
$$

Where $r$ and $t$ take any value but preferable are just 1,2,3,4. The following four TL-moments are obtained by using (10)

$$
\begin{aligned}
& \lambda_{1}^{(1)}=E\left(X_{2: 3}\right)=6 \int_{0}^{1} x(F) F(1-F) d F \\
& \lambda_{2}^{(1)}=\frac{1}{2} E\left(X_{3: 4}-X_{2: 4}\right)=6 \int_{0}^{1} x(F) F(1-F)(2 F-1) d F \\
& \lambda_{3}^{(1)}=\frac{1}{3} E\left(X_{4: 5}-2 X_{3: 5}+X_{2: 5}\right)=\frac{20}{3} \int_{0}^{1} x(F) F(1-F)\left(5 F^{2}-5 F+1\right) d F \\
& \lambda_{4}^{(1)}=\frac{1}{4} E\left(X_{5: 6}-3 X_{4: 6}+3 X_{3: 6}-X_{2: 6}\right)=\frac{15}{2} \int_{0}^{1} x(F) F(1-F)\left(14 F^{3}-21 F^{2}+9 F-1\right) d F
\end{aligned}
$$

The population TL-skewness and TL-kurtosis are $\tau_{3}^{(1)}=\lambda_{3}^{(1)} / \lambda_{2}^{(1)}$ and $\tau_{4}^{(1)}=\lambda_{4}^{(1)} / \lambda_{2}^{(1)}$ respectively. TL-moment can be estimated from a sample as linear combination of order statistics. Following are the sample TL-moment by [6]

$$
\begin{aligned}
& l_{1}^{(1)}=\frac{3 !(n-3) !}{n !} \sum_{j=2}^{n-1} C_{1}^{j-1} C_{1}^{n-j} X_{J: n} \\
& l_{2}^{(1)}=\frac{4 !(n-4) !}{2 n !} \sum_{j=2}^{n-1}\left[C_{2}^{j-1} C_{1}^{n-j}-C_{1}^{j-1} C_{2}^{n-j}\right] X_{j: n} \\
& l_{3}^{(1)}=\frac{5 !(n-5) !}{3 n !} \sum_{j=2}^{n-1}\left[C_{3}^{j-1} C_{1}^{n-j}-2 C_{2}^{j-1} C_{2}^{n-j}+C_{1}^{j-1} C_{3}^{n-j}\right] X_{J: n} \\
& l_{4}^{(1)}=\frac{6 !(n-6) !}{4 n !} \sum_{j=2}^{n-1}\left[C_{4}^{j-1} C_{1}^{n-j}-3 C_{3}^{j-1} C_{2}^{n-j}+3 C_{2}^{j-1} C_{3}^{n-j}-C_{1}^{j-1} C_{4}^{n-j}\right] X_{j: n}
\end{aligned}
$$

The sample TL-skewness and TL-kurtosis are $t_{3}^{(1)}=l_{3}^{(1)} / l_{2}^{(1)}$ and $t_{4}^{(1)}=l_{4}^{(1)} / l_{2}^{(1)}$ respectively.

\subsection{Singh Maddala distribution}

The family of distributions proposed by [15], whose core distribution was the generalized beta distribution, became popular distribution for fitting the distribution on income or consumption expenditure (McDonald, 1984). The probability distribution function is defined as $f(x)=a q x^{a-1} /\left[b^{a}\left\{1+(x / b)^{a}\right\}^{q+1}\right]$. Where $a$ is the shape parameter $(a>0), b$ is the scale parameter $(b>0), q$ is the shape parameter $(k>0)$ and $0 \leq x<+\infty$. And its cumulative distribution function is $F(x)=\left[1-\left(1+(x / b)^{a}\right)^{-q}\right]$, the mean of the distribution is $E(x)=b \Gamma(1+1 / a) \Gamma(q-1 / a) / \Gamma q$ and the rth moment about zero mean is

$$
E\left(x^{r}\right)=b^{r} \Gamma(1+r / a)(q-r / a) / \Gamma q
$$

Almost all the well-known statistical distributions are considered for the description of income distribution but this distribution provides the best fit, [6]. It has been found by [4] that the SMD was better fit than those of Beta type-I or Generalized Gamma distributions when analysed for Japanese income data.

\section{L-moments and TL-moments for the SMD}

To date, according to our knowledge there is no derivation of the L-moments and TL-moments the SMD in the literature. In this section, we derive these moments using the general rule to get the L-moments and TL-moments of any distribution. The first four L-moments and TL-moments are derived as follows: 


\subsection{L-Moments for SMD}

We use $X$ a real value random variable and let $X_{1: n} \leq X_{2: n} \leq X_{3: n} \leq \ldots \leq X_{n: n}$ be the order statistics. The expectation of the order statistics is as

$$
E\left(X_{r: n}\right)=\frac{n !}{(r-1) !(n-r)} \int x[F(x)]^{r-1}[1-F(x)]^{n-r} d F(x)
$$

Substituting the distribution function of the SMD in (20) we obtain

$$
E\left(X_{r: n}\right)=\frac{n ! a q}{(r-1) !(n-r) !} \int_{0}^{\infty}\left(x_{r: n} / b\right)^{a}\left\{1+\left(x_{r: n} / b\right)^{a}\right\}^{-q(n-r+1)-1}\left[1-\left\{1+\left(x_{r: n} / b\right)^{a}\right\}^{-q}\right]^{r-1} d x_{r: n}
$$

And the quantile function of the SMD is

$$
x(F)=b\left([1-F]^{-\frac{1}{q}}-1\right)^{\frac{1}{a}}
$$

Now using (2) to (5) relationship, the first four L-moments for the SMD are derived. The first L-moment $\left(\lambda_{1}\right)$ is always equal to the mean of the moment, so

$$
\lambda_{1}=E(X)=b \Gamma(1+1 / a) \Gamma(q-1 / a) / \Gamma q
$$

The second L-moment $\left(\lambda_{2}\right)$ is defined as

$$
\lambda_{2}=b \Gamma(1+1 / a)\{\Gamma(q-1 / a) / \Gamma q-\Gamma(2 q-1 / a) / \Gamma 2 q\}
$$

The third L-moment $\left(\lambda_{3}\right)$ is defined as

$$
\lambda_{3}=b \Gamma(1+1 / a)\{\Gamma(q-1 / a) / \Gamma(q)-3 \Gamma(2 q-1 / a) / \Gamma(2 q)+2 \Gamma(3 q-1 / a) / \Gamma(3 q)\}
$$

The fourth L-moment $\left(\lambda_{4}\right)$ is derived and get this result

$$
\lambda_{4}=b \Gamma(1+1 / a)\left\{\frac{\Gamma(q-1 / a)}{\Gamma q}-\frac{6 \Gamma(2 q-1 / a)}{\Gamma(2 q)}+\frac{10 \Gamma(3 q-1 / a)}{\Gamma(3 q)}-\frac{5 \Gamma(4 q-1 / a)}{\Gamma(4 q)}\right\}
$$

Equating the population moments with sample moments and after simplification we can estimate the parameter of the distribution.

\subsection{TL-Moments for the SMD}

The TL-Moments of the SMD distribution are derived by using the (11) to (15)

$$
\begin{aligned}
& \lambda_{1}^{(1)}=b \Gamma(1+1 / a)\{3 \Gamma(2 q-1 / a) \Gamma(2 q)-2 \Gamma(3 q-1 / a) / \Gamma(3 q)\} \\
& \lambda_{2}^{(1)}=3 b \Gamma(1+1 / a)\{\Gamma(2 q-1 / a) / \Gamma(2 q)-2 \Gamma(3 q-1 / a) / \Gamma(3 q)+\Gamma(4 q-1 / a) / \Gamma 4 q\} \\
& \lambda_{3}^{(1)}=\frac{10 b}{3} \Gamma(1+1 / a)\left\{\frac{\Gamma(2 q-1 / a)}{\Gamma(2 q)}-\frac{4 \Gamma(3 q-1 / a)}{\Gamma(3 q)}+\frac{5 \Gamma(4 q-1 / a)}{\Gamma(4 q)}-\frac{2 \Gamma(5 q-1 / a)}{\Gamma(5 q)}\right\} \\
& \lambda_{4}^{(1)}=15 b \Gamma\left(1+\frac{1}{a}\right)\left\{\frac{\Gamma(2 q-1 / a)}{4 \Gamma(2 q)}-\frac{5 \Gamma(3 q-1 / a)}{3 \Gamma(3 q)}+\frac{15 \Gamma(4 q-1 / a)}{4 \Gamma(4 q)}-\frac{7 \Gamma(5 q-1 / a)}{2 \Gamma(5 q)}+\frac{7 \Gamma(6 q-1 / a)}{6 \Gamma(6 q)}\right\}
\end{aligned}
$$

Form these results we can obtain the TL-moments estimates.

\subsection{L and TL coefficient of variation, skewness and kurtosis}

The $L-C V=\lambda_{2} / \lambda_{1}$ is the L-moment $C V$ and its defined range is $0<L-C V<1, \quad 0<L-C S\left(\left|\lambda_{3} / \lambda_{2}\right|\right)<1$ is measure of skewness (asymmetry) with the range 0 and 1 . And measure of kurtosis which is related to the asymmetry and peakedness of any distribution lies in $\left(5 \tau_{3}^{2}-1\right) / 4 \leq L-C K\left(\lambda_{4} / \lambda_{2}\right)<1$ according to [9]. The population CV, the CS and the $\mathrm{CK}$ for the $\mathrm{L}$ Moments of the SMD are as follows:

$$
L-C V=1-\frac{\Gamma(2 q-1 / a) \Gamma q}{\Gamma(q-1 / a) \Gamma 2 q}
$$




$$
\begin{aligned}
L-C S & =\frac{\{\Gamma(q-1 / a) / \Gamma q-3 \Gamma(2 q-1 / a) / \Gamma 2 q+2 \Gamma(3 q-1 / a) / \Gamma 3 q\}}{\{\Gamma(q-1 / a) / \Gamma q-\Gamma(2 q-1 / a) / \Gamma 2 q\}} \\
L-C K & =\frac{\{\Gamma(q-1 / a) / \Gamma q-6 \Gamma(2 q-1 / a) / \Gamma 2 q+10 \Gamma(3 q-1 / a) / \Gamma 3 q-5 \Gamma(4 q-1 / a) / \Gamma 4 q\}}{\{\Gamma(q-1 / a) / \Gamma q-\Gamma(2 q-1 / a) / \Gamma 2 q\}}
\end{aligned}
$$

The population $\mathrm{CV}$, the $\mathrm{CS}$ and the $\mathrm{CK}$ for the TL Moments of the SMD are as follows:

$$
\begin{aligned}
& T L-C V=\frac{3\{\Gamma(2 q-1 / a) / \Gamma 2 q-2 \Gamma(3 q-1 / a) / \Gamma 3 q+\Gamma(4 q-1 / a) / \Gamma 4 q\}}{\{3 \Gamma(2 q-1 / a) \Gamma 2 q-2 \Gamma(3 q-1 / a) / \Gamma 3 q\}} \\
& T L-C S= \frac{10\left\{\frac{\Gamma(2 q-1 / a)}{\Gamma(2 q)}-\frac{4 \Gamma(3 q-1 / a)}{\Gamma(3 q)}+\frac{5 \Gamma(4 q-1 / a)}{\Gamma(4 q)}-\frac{2 \Gamma(5 q-1 / a)}{\Gamma(5 q)}\right\}}{9\left\{\frac{\Gamma(2 q-1 / a)}{\Gamma(2 q)}-\frac{2 \Gamma(3 q-1 / a)}{\Gamma(3 q)}+\frac{\Gamma(4 q-1 / a)}{\Gamma(4 q)}\right\}} \\
& T L-C K=\frac{5\left\{\frac{\Gamma(2 q-1 / a)}{4 \Gamma(2 q)}-\frac{5 \Gamma(3 q-1 / a)}{3 \Gamma(3 q)}+\frac{15 \Gamma(4 q-1 / a)}{4 \Gamma(4 q)}-\frac{7 \Gamma(5 q-1 / a)}{2 \Gamma(5 q)}+\frac{7 \Gamma(6 q-1 / a)}{6 \Gamma(6 q)}\right\}}{\{\Gamma(2 q-1 / a) / \Gamma 2 q-2 \Gamma(3 q-1 / a) / \Gamma 3 q+\Gamma(4 q-1 / a) / \Gamma 4 q\}}
\end{aligned}
$$

The general ranges of the TL-skewness and kurtosis is not found in the literature.

\section{Monte Carlo simulation base study}

In this section, we present empirical analysis which is based on the simulated data to compare the properties of the method of moments, L-Moments and TL-moments estimators of the SMD distribution with respect to their biases, RMSEs, CV, CS and the CK. The sets of data is simulated using the MATLAB-7 software taking different sample sizes e.g 50, 100, 500 and 1000 and assuming different value of each parameter. The SMD is a three parameter distribution, $a$ and $q$ are shape parameter and $b$ is the scale parameter. In simulation, various values for the parameter $a, b$ and $q$ are assumed to estimate the scale parameter. We take the similar parametric values as [17] taken in their study of beta distribution. We also take a higher value of scale parameter as 10. In simulation experiment each sample was repeated 10,000 times to obtain the precision and accuracy.

It is observed from Table A.1 to A.4 that the all the estimators underestimate the parameter $b$, as the most of the biases are negative but very close to the true parameter. One can observe in the case of small sample size and small value of parameters TL-moments provides minimum RMSEs, but most of the cases the RMSEs for L-moments are quite small in the case of large sample size and large values of parameters. Therefore it is expected that the L-moments provide more precise estimates of the scale parameters for the SMD.

The CV, CS and CK using the equations from (31) to (36) are computed and presented in the Table A.5, assuming different parameters values. From Table A.5 we can observe that the coefficients of TL-moments are relatively small than the conventional method of moments, the L-moments coefficients. Both the L and TL-moments provide good estimates for a random variable following the SMD. But we prefer the TL-moments since it has the smallest RMSEs. We also observe that the shape parameters change the values of coefficients but for the different scale parameters the coefficients remain constant. It also verifies that the derived expressions are up-to the mark, as scale parameter does not affect the skewness and kurtosis of the distribution.

\section{Conclusion}

We have derived L-moments and TL moments for the SMD and compare their performance on the basis of bias and the RMSEs for the scale parameter estimation and found that overall L-moment method of estimation is relatively efficient. L-moments provide the best results for sample size above 50, but for sample size less than 50 (approximately) TLmoments give almost unbiased results with minimum RMSEs. In term of distribution fitting TL-moments also provide superior fitting. 


\section{References}

[1] Abdul-Moniem, I., and Youssef M. Selim, "TL-moments and L-moments estimation for the generalized Pareto distribution" Applied Mathematical Sciences. Vol.3, No.1 (2009), pp. 43-52.

[2] Abdul-Moniem, Ibrahim B. "L-moments and TL-moments estimation for the exponential distribution." Far East Journal of Theoretical Statistics, Vol.23, No.1, (2007), pp.51-61.

[3] Ahmad, Ummi Nadiah, Ani Shabri, and Zahrahtul Amani Zakaria, "TL-moments and L-moments Estimation of the Generalized Logistic Distribution", Journal of Mathematics Research,Vol. 3, No.1, (2011), pp.97-106.

[4] Asquith, William H., "L-moments and TL-moments of the generalized lambda distribution", Computational Statistics \& Data Analysis, Vol.51, No.9, (2007), pp. 4484-4496.

[5] Atoda, Naosumi, Surura, T. and Tachibanaki, T., "Statistical Inference of Functional Forms for Income Distribution". manuscript, (1980), Kyoto University.

[6] Dhongde, Shatakshee, and Camelia Minoiu, "Global poverty estimates: Present and future", Society for the Study of Economic Inequality, Working Paper No 181 (2010).

[7] Elamir, Elsayed AH, and Allan H. Seheult, "Trimmed L-moments", Computational Statistics \& Data Analysis, Vol. 43, No.3, (2003), pp. 299314.

[8] Greenwood, J. A., Landwehr, J. M., Matalas, N. C., \& Wallis, J. R., "Probability weighted moments: definition and relation to parameters of several distributions expressable in inverse form", Water Resources Research, Vol.15, No.5, (1979), pp.1049-1054.

[9] Hosking, J. R. M., and J. R. Wallis, "A comparison of unbiased and plotting-position estimators of L moments", Water Resources Research, Vol. 31, No.8, (1995), pp.2019-2025.

[10] Hosking, Jonathan RM., "L-moments: analysis and estimation of distributions using linear combinations of order statistics", Journal of the Royal Statistical Society. Series B (Methodological), (1990), pp.105-124.

[11] Kleiber, Christian, "Dagum vs. Singh-Maddala income distributions", Economics Letters, Vol. 53, No.3, (1996), pp.265-268.

[12] Kundu, Debasis, and Mohammad Z. Raqab, "Generalized Rayleigh distribution: different methods of estimations", Computational statistics \& data analysis, Vol. 49, No.1, (2005), pp.187-200.

[13] McDonald, James B., "Some generalized functions for the size distribution of income", Econometrica: Journal of the Econometric Society, (1984), pp.647-663.

[14] Shahzad, M. N, and Asghar, Z., "Comparing TL-Moments, L-Moments and Conventional Moments of Dagum Distribution by Simulated data", Revista Colombiana de Estadística, Vol. 36, No.1, (2013), pp.79-93.

[15] Singh, S.K., and G.S. Maddala, "A Function for the size distribution of income”, Econometrica,Vol. 44, (1976), pp.963-970.

[16] Vogel, Richard M., and Neil M. Fennessey, "L moment diagrams should replace product moment diagrams", Water Resources Research, Vol. 29, No.6, (1993), pp.1745-1752.

[17] Ye, Yuan, Broderick O. Oluyede, and Mavis Pararai, "Weighted generalized beta distribution of the second kind and related distributions", Journal of Statistical and Econometric Methods, Vol.1, No.1, (2012), pp.13-31.

[18] Zhu, Feng."A nonparametric analysis of the shape dynamics of the US personal income distribution", BIS Working Papers, 184, (2005), pp.1962-2000". 


\section{Appendix A}

Table A.1: Biases and RMSEs of the parameter estimations for different types of estimators assuming SMD for $b$ when $b=2.5$

\begin{tabular}{|c|c|c|c|c|c|c|c|c|}
\hline \multicolumn{3}{|c|}{ Parameters } & \multicolumn{3}{|c|}{$\mathrm{n}=50$} & \multicolumn{3}{|c|}{$\mathrm{n}=100$} \\
\hline$a$ & $q$ & & MME & LME & TLME & MME & LME & TLME \\
\hline \multirow[t]{6}{*}{2.5} & \multirow[t]{2}{*}{2.5} & Bias & -0.0645 & -0.0002 & -0.0016 & -0.0370 & -0.0004 & 0.0003 \\
\hline & & RMSEs & 0.4567 & 0.3497 & 0.3442 & 0.3268 & 0.2487 & 0.2430 \\
\hline & \multirow[t]{2}{*}{3.5} & Bias & -0.0494 & 0.0016 & 0.0032 & -0.0318 & -0.0043 & -0.0045 \\
\hline & & RMSEs & 0.3571 & 0.3069 & 0.3264 & 0.2543 & 0.2141 & 0.2255 \\
\hline & \multirow[t]{2}{*}{5} & Bias & -0.0034 & -0.0022 & -0.0034 & -0.0222 & 0.0004 & 0.0011 \\
\hline & & RMSEs & 0.3182 & 0.2893 & 0.3191 & 0.2222 & 0.2010 & 0.2204 \\
\hline \multirow[t]{6}{*}{3.5} & \multirow[t]{2}{*}{2.5} & Bias & -0.0535 & -0.0050 & -0.0035 & -0.0226 & 0.0021 & 0.0017 \\
\hline & & RMSEs & 0.3422 & 0.2991 & 0.3215 & 0.2460 & 0.2123 & 0.2123 \\
\hline & \multirow[t]{2}{*}{3.5} & Bias & -0.0427 & -0.0014 & -0.0036 & -0.0215 & -0.0002 & -0.0010 \\
\hline & & RMSEs & 0.2933 & 0.2741 & 0.3066 & 0.2086 & 0.1943 & 0.2164 \\
\hline & \multirow[t]{2}{*}{5} & Bias & -0.0417 & -0.0011 & 0.0011 & -0.0196 & 0.0002 & 0.0012 \\
\hline & & RMSEs & 0.2685 & 0.2634 & 0.3041 & 0.1899 & 0.1846 & 0.2118 \\
\hline \multirow[t]{6}{*}{5} & \multirow[t]{2}{*}{2.5} & Bias & -0.0444 & -0.0018 & -0.0021 & -0.0209 & 0.0002 & -0.0002 \\
\hline & & RMSEs & 0.2971 & 0.2815 & 0.3109 & 0.2067 & 0.1944 & 0.2145 \\
\hline & \multirow[t]{2}{*}{3.5} & Bias & -0.0422 & -0.0033 & -0.0045 & -0.0208 & -0.0010 & -0.0006 \\
\hline & & RMSEs & 0.2680 & 0.2654 & 0.3074 & 0.1885 & 0.1860 & 0.2128 \\
\hline & \multirow[t]{2}{*}{5} & Bias & -0.0325 & 0.0052 & 0.0033 & -0.0213 & -0.0022 & -0.0020 \\
\hline & & RMSEs & 0.2566 & 0.2594 & 0.3029 & 0.1810 & 0.1816 & 0.2103 \\
\hline & \multicolumn{2}{|c|}{ Parameters } & \multicolumn{3}{|c|}{$\mathrm{n}=500$} & \multicolumn{3}{|c|}{$\mathrm{n}=1000$} \\
\hline$a$ & $q$ & & MME & LME & TLME & MME & LME & TLME \\
\hline \multirow[t]{6}{*}{2.5} & \multirow[t]{2}{*}{2.5} & Bias & -0.0051 & 0.0009 & 0.00003 & -0.0039 & -0.0002 & -0.0004 \\
\hline & & RMSEs & 0.1612 & 0.1107 & 0.1069 & 0.0754 & 0.0783 & 0.0754 \\
\hline & \multirow[t]{2}{*}{3.5} & Bias & -0.0058 & -0.0003 & 0.0001 & -0.0040 & -0.0011 & -0.0010 \\
\hline & & RMSEs & 0.1202 & 0.0983 & 0.1025 & 0.0843 & 0.0687 & 0.0717 \\
\hline & \multirow[t]{2}{*}{5} & Bias & -0.0045 & -.00001 & -.00005 & -0.0027 & -0.0003 & -0.0002 \\
\hline & & RMSEs & 0.1023 & 0.0908 & 0.0978 & 0.0712 & 0.0631 & 0.0684 \\
\hline \multirow[t]{6}{*}{3.5} & \multirow[t]{2}{*}{2.5} & Bias & -0.0056 & -0.0004 & 0.00001 & -0.0027 & -0.0002 & 0.0001 \\
\hline & & RMSEs & 0.1138 & 0.0953 & 0.1004 & 0.0815 & 0.0670 & 0.0699 \\
\hline & \multirow[t]{2}{*}{3.5} & Bias & -0.0043 & 0.0002 & 0.0009 & -0.0024 & -0.0005 & -0.0010 \\
\hline & & RMSEs & 0.0958 & 0.0876 & 0.0958 & 0.0668 & 0.0612 & 0.0673 \\
\hline & 5 & Bias & -0.0044 & -0.0006 & -0.0009 & -0.0026 & -0.0007 & -0.0008 \\
\hline & & RMSEs & 0.0836 & 0.0813 & 0.0939 & 0.0602 & 0.0582 & 0.0665 \\
\hline 5 & 2.5 & Bias & -0.0050 & -0.0006 & -0.0001 & -0.0038 & -0.0017 & -0.0018 \\
\hline & & RMSEs & 0.0945 & 0.0884 & 0.0974 & 0.0665 & 0.0620 & 0.0679 \\
\hline & 3.5 & Bias & -0.0043 & -0.0003 & -0.0002 & -0.0019 & 0.0001 & 0.0007 \\
\hline & & RMSEs & 0.0844 & 0.0830 & 0.0943 & 0.0599 & 0.0588 & 0.0667 \\
\hline & 5 & Bias & -0.0040 & -0.0002 & 0.0002 & -0.0029 & -0.0011 & -0.0014 \\
\hline & & RMSEs & 0.0801 & 0.0807 & 0.0934 & 0.0568 & 0.0573 & 0.0665 \\
\hline
\end{tabular}

MME: Method of moment estimator, LME: L-moment estimator, TLME: TL-moment estimator 
Table A.2: Biases and RMSEs of the parameter estimations for different types of estimators assuming SMD for $\mathrm{b}$ when $b=3.5$

\begin{tabular}{|c|c|c|c|c|c|c|c|c|}
\hline \multicolumn{3}{|c|}{ Parameters } & \multicolumn{3}{|c|}{$\mathrm{n}=50$} & \multicolumn{3}{|c|}{$\mathrm{n}=100$} \\
\hline$a$ & $q$ & & MME & LME & TLME & MME & LME & TLME \\
\hline \multirow[t]{6}{*}{2.5} & \multirow[t]{2}{*}{2.5} & Bias & -0.0896 & 0.0022 & 0.0046 & -0.0479 & 0.0012 & 0.0024 \\
\hline & & RMSEs & 0.6254 & 0.4854 & 0.4810 & 0.4653 & 0.3447 & 0.3347 \\
\hline & \multirow[t]{2}{*}{3.5} & Bias & -0.0701 & 0.0016 & 0.0017 & -0.0409 & -0.0048 & -0.0057 \\
\hline & & RMSEs & 0.5065 & 0.4349 & 0.4584 & 0.3680 & 0.3074 & 0.3200 \\
\hline & \multirow[t]{2}{*}{5} & Bias & -0.0620 & -0.0011 & -0.0042 & -0.0382 & -0.0059 & -0.0052 \\
\hline & & RMSEs & 0.4364 & 0.3964 & 0.4377 & 0.3103 & 0.2805 & 0.3099 \\
\hline \multirow[t]{6}{*}{3.5} & \multirow[t]{2}{*}{2.5} & Bias & -0.0686 & -0.0010 & -0.0019 & -0.0319 & 0.0014 & -0.0019 \\
\hline & & RMSEs & 0.4849 & 0.4228 & 0.4483 & 0.3482 & 0.2971 & 0.3125 \\
\hline & \multirow[t]{2}{*}{3.5} & Bias & -0.0689 & -0.0094 & -0.0102 & -0.0349 & -0.0041 & -0.0032 \\
\hline & & RMSEs & 0.4114 & 0.3858 & 0.4313 & 0.2870 & 0.2689 & 0.3014 \\
\hline & \multirow[t]{2}{*}{5} & Bias & -0.0614 & -0.0056 & -0.0036 & -0.0290 & -0.0011 & 0.0003 \\
\hline & & RMSEs & 0.3760 & 0.3678 & 0.4258 & 0.2654 & 0.2586 & 0.2967 \\
\hline \multirow[t]{6}{*}{5} & \multirow[t]{2}{*}{2.5} & Bias & -0.0596 & -0.0010 & -0.0031 & -0.0276 & 0.0016 & 0.0015 \\
\hline & & RMSEs & 0.4127 & 0.3923 & 0.4346 & 0.2915 & 0.2739 & 0.3046 \\
\hline & \multirow[t]{2}{*}{3.5} & Bias & -0.0504 & 0.0027 & -0.0022 & -0.0265 & 0.0003 & -0.0016 \\
\hline & & RMSEs & 0.3723 & 0.3686 & 0.4228 & 0.2635 & 0.2596 & 0.2963 \\
\hline & \multirow[t]{2}{*}{5} & Bias & -0.0540 & -0.0005 & -0.0001 & -0.0232 & 0.0031 & 0.0007 \\
\hline & & RMSEs & 0.3608 & 0.3641 & 0.4256 & 0.2497 & 0.2522 & 0.2928 \\
\hline & \multicolumn{2}{|c|}{ Parameters } & & $\mathrm{n}=500$ & & & $n=1000$ & \\
\hline$a$ & $q$ & & MME & LME & TLME & MME & LME & TLME \\
\hline \multirow[t]{6}{*}{2.5} & \multirow[t]{2}{*}{2.5} & Bias & -0.0085 & -.00003 & -0.0012 & -0.0052 & 0.0003 & 0.0004 \\
\hline & & RMSEs & 0.2297 & 0.1563 & 0.1494 & 0.1649 & 0.1113 & 0.1064 \\
\hline & \multirow[t]{2}{*}{3.5} & Bias & -0.0045 & 0.0023 & 0.0020 & -0.0047 & -0.0002 & -0.0001 \\
\hline & & RMSEs & 0.1693 & 0.1379 & 0.1431 & 0.1186 & 0.0973 & 0.1019 \\
\hline & \multirow[t]{2}{*}{5} & Bias & -0.0061 & -.00004 & -0.0002 & -0.0035 & -0.0004 & -0.0001 \\
\hline & & RMSEs & 0.1403 & 0.1248 & 0.1362 & 0.0999 & 0.0885 & 0.0964 \\
\hline \multirow[t]{6}{*}{3.5} & \multirow[t]{2}{*}{2.5} & Bias & -0.0062 & 0.0004 & -.00004 & -0.0038 & -0.0007 & -0.0009 \\
\hline & & RMSEs & 0.1578 & 0.1316 & 0.1391 & 0.1126 & 0.0926 & 0.0971 \\
\hline & \multirow[t]{2}{*}{3.5} & Bias & -0.0066 & -0.0010 & -0.0020 & -0.0039 & -0.0007 & -0.0002 \\
\hline & & RMSEs & 0.1327 & 0.1220 & 0.1343 & 0.0937 & 0.0861 & 0.0948 \\
\hline & \multirow[t]{2}{*}{5} & Bias & -0.0053 & 0.0001 & 0.0003 & -0.0045 & -0.0018 & -0.0020 \\
\hline & & RMSEs & 0.1182 & 0.1147 & 0.1313 & 0.0843 & 0.0816 & 0.0927 \\
\hline \multirow[t]{6}{*}{5} & 2.5 & Bias & -0.0069 & -0.0005 & .00006 & -0.0027 & 0.0003 & 0.0006 \\
\hline & & RMSEs & 0.1316 & 0.1221 & 0.1333 & 0.0927 & 0.0862 & 0.0948 \\
\hline & 3.5 & Bias & -0.0034 & 0.0017 & 0.0015 & -0.0022 & 0.0005 & 0.0005 \\
\hline & & RMSEs & 0.1152 & 0.1151 & 0.1312 & 0.0847 & 0.0830 & 0.0937 \\
\hline & 5 & Bias & -0.0060 & -0.0006 & -0.0003 & -0.0031 & -0.0004 & -0.0001 \\
\hline & & RMSEs & 0.1116 & 0.1122 & 0.1303 & 0.0792 & 0.0797 & 0.0925 \\
\hline
\end{tabular}


Table A.3: Biases and RMSEs of the parameter estimations for different types of estimators assuming SMD for $\mathrm{b}$ when $b=5$

\begin{tabular}{|c|c|c|c|c|c|c|c|c|}
\hline \multicolumn{3}{|c|}{ Parameters } & \multicolumn{3}{|c|}{$\mathrm{n}=50$} & \multicolumn{3}{|c|}{$\mathrm{n}=100$} \\
\hline $\mathrm{a}$ & $q$ & & MME & LME & TLME & MME & LME & TLME \\
\hline \multirow[t]{6}{*}{2.5} & \multirow[t]{2}{*}{2.5} & Bias & -0.1247 & 0.0074 & 0.0054 & -0.0795 & -0.0035 & -0.0001 \\
\hline & & RMSEs & 0.9049 & 0.7027 & 0.6920 & 0.6469 & 0.4887 & 0.4805 \\
\hline & \multirow[t]{2}{*}{3.5} & Bias & -0.0996 & -0.0019 & -0.0076 & -0.0528 & -0.0013 & -0.0007 \\
\hline & & RMSEs & 0.7260 & 0.6181 & 0.6502 & 0.5265 & 0.4393 & 0.4588 \\
\hline & \multirow[t]{2}{*}{5} & Bias & -0.0962 & -0.0962 & -0.0124 & -0.0507 & -0.0058 & -0.0061 \\
\hline & & RMSEs & 0.6166 & 0.5616 & 0.6176 & 0.4481 & 0.4047 & 0.4439 \\
\hline \multirow{6}{*}{3.5} & \multirow[t]{2}{*}{2.5} & Bias & -0.1018 & -0.0061 & -0.0101 & -0.0451 & 0.0016 & -0.0026 \\
\hline & & RMSEs & 0.6879 & 0.6015 & 0.6371 & 0.5018 & 0.4248 & 0.4466 \\
\hline & \multirow[t]{2}{*}{3.5} & Bias & -0.0937 & -0.0105 & -0.0159 & -0.0378 & 0.0045 & 0.0053 \\
\hline & & RMSEs & 0.5907 & 0.5554 & 0.6179 & 0.4201 & 0.3901 & 0.4309 \\
\hline & \multirow[t]{2}{*}{5} & Bias & -0.0808 & -0.0017 & -0.0020 & -0.0391 & -0.0004 & -0.0014 \\
\hline & & RMSEs & 0.5389 & 0.5281 & 0.6078 & 0.3799 & 0.3698 & 0.4258 \\
\hline \multirow[t]{6}{*}{5} & \multirow[t]{2}{*}{2.5} & Bias & -0.0875 & -0.0045 & -0.0051 & -0.0464 & -0.0037 & -0.0036 \\
\hline & & RMSEs & 0.5914 & 0.5611 & 0.6207 & 0.4152 & 0.3917 & 0.4342 \\
\hline & \multirow[t]{2}{*}{3.5} & Bias & -0.0752 & 0.0024 & 0.0012 & -0.0432 & -0.0036 & -0.0027 \\
\hline & & RMSEs & 0.5409 & 0.5344 & 0.6110 & 0.3821 & 0.3771 & 0.4301 \\
\hline & \multirow[t]{2}{*}{5} & Bias & -0.0750 & 0.0016 & 0.0047 & -0.0386 & -0.0003 & 0.00058 \\
\hline & & RMSEs & 0.5133 & 0.5199 & 0.6120 & 0.3583 & 0.3613 & 0.4231 \\
\hline & \multicolumn{2}{|c|}{ Parameters } & & $\mathrm{n}=500$ & & & $\mathrm{n}=1000$ & \\
\hline $\mathrm{a}$ & $q$ & & MME & LME & TLME & MME & LME & TLME \\
\hline \multirow[t]{6}{*}{2.5} & \multirow[t]{2}{*}{2.5} & Bias & -0.0162 & 0.0015 & 0.0045 & -0.0078 & -0.0017 & -0.0026 \\
\hline & & RMSEs & 0.3183 & 0.2176 & 0.2121 & 0.2338 & 0.1558 & 0.1515 \\
\hline & \multirow[t]{2}{*}{3.5} & Bias & -0.0111 & -0.0013 & -0.0020 & -0.0060 & -0.0003 & 0.0005 \\
\hline & & RMSEs & 0.2371 & 0.1926 & 0.2023 & 0.1694 & 0.1366 & 0.1423 \\
\hline & \multirow[t]{2}{*}{5} & Bias & -0.0091 & -.00003 & -0.0001 & -0.0049 & -0.0006 & -0.0009 \\
\hline & & RMSEs & 0.2046 & 0.1816 & 0.1957 & 0.1431 & 0.1269 & 0.1382 \\
\hline \multirow[t]{6}{*}{3.5} & \multirow[t]{2}{*}{2.5} & Bias & -0.0117 & -0.0014 & -0.0012 & -0.0078 & -0.0023 & -0.0021 \\
\hline & & RMSEs & 0.2266 & 0.1883 & 0.1972 & 0.1598 & 0.1333 & 0.1404 \\
\hline & \multirow[t]{2}{*}{3.5} & Bias & -0.0085 & 0.0003 & 0.0007 & -0.0041 & 0.0002 & 0.0003 \\
\hline & & RMSEs & 0.1869 & 0.1730 & 0.1925 & 0.1354 & 0.1238 & 0.1360 \\
\hline & \multirow[t]{2}{*}{5} & Bias & -0.0074 & 0.0005 & 0.0016 & -0.0042 & -0.0005 & -0.0011 \\
\hline & & RMSEs & 0.1704 & 0.1654 & 0.1895 & 0.1201 & 0.1159 & 0.1319 \\
\hline \multirow[t]{6}{*}{5} & 2.5 & Bias & -0.0122 & -0.0035 & -0.0042 & -0.0048 & -.00028 & 0.00003 \\
\hline & & RMSEs & 0.1869 & 0.1742 & 0.1901 & 0.1323 & 0.1231 & 0.1348 \\
\hline & 3.5 & Bias & -0.0076 & 0.0004 & 0.00155 & -0.0041 & -0.0004 & -0.0010 \\
\hline & & RMSEs & 0.1705 & 0.1676 & 0.1909 & 0.1200 & 0.1174 & 0.1328 \\
\hline & 5 & Bias & -0.0092 & -0.0014 & -0.0005 & -0.0036 & 0.0002 & 0.00034 \\
\hline & & RMSEs & 0.1623 & 0.1635 & 0.1896 & 0.1146 & 0.1150 & 0.1325 \\
\hline
\end{tabular}


Table A.4: Biases and RMSEs of the parameter estimationrs for different types of estimators assuming SMD for $b$ when $b=10$

\begin{tabular}{|c|c|c|c|c|c|c|c|c|}
\hline \multicolumn{3}{|c|}{ Parameters } & \multicolumn{3}{|c|}{$\mathrm{n}=50$} & \multicolumn{3}{|c|}{$\mathrm{n}=100$} \\
\hline $\mathrm{a}$ & $q$ & & MME & LME & TLME & MME & LME & TLME \\
\hline \multirow[t]{6}{*}{2.5} & \multirow[t]{2}{*}{2.5} & Bias & -0.2562 & 0.0065 & 0.0132 & -0.1483 & -0.0074 & -0.0070 \\
\hline & & RMSEs & 1.7869 & 1.3870 & 1.3742 & 1.3197 & 0.9804 & 0.9584 \\
\hline & \multirow[t]{2}{*}{3.5} & Bias & -0.1635 & -0.0045 & -0.0097 & -0.1153 & -0.0129 & -0.0188 \\
\hline & & RMSEs & 1.4408 & 1.2335 & 1.2969 & 1.0372 & 0.8624 & 0.8994 \\
\hline & \multirow[t]{2}{*}{5} & Bias & -0.1772 & -0.0032 & -0.0122 & -0.0883 & -0.0020 & -0.0058 \\
\hline & & RMSEs & 1.2469 & 1.1327 & 1.2507 & 0.8985 & 0.8096 & 0.8840 \\
\hline \multirow[t]{6}{*}{3.5} & \multirow[t]{2}{*}{2.5} & Bias & -0.1964 & -0.0037 & -0.0100 & -0.0901 & 0.0065 & 0.0053 \\
\hline & & RMSEs & 1.3818 & 1.2082 & 1.2771 & 0.9798 & 0.8342 & 0.8827 \\
\hline & \multirow[t]{2}{*}{3.5} & Bias & -0.1818 & -0.0133 & -0.0187 & -0.0862 & -0.0038 & -0.0091 \\
\hline & & RMSEs & 1.1858 & 1.1119 & 1.2317 & 0.8413 & 0.7762 & 0.8647 \\
\hline & \multirow[t]{2}{*}{5} & Bias & -0.1586 & -0.0010 & 0.0004 & -0.0839 & -0.0022 & 0.0060 \\
\hline & & RMSEs & 1.0750 & 1.0537 & 1.2175 & 0.7585 & 0.7381 & 0.8469 \\
\hline \multirow[t]{6}{*}{5} & \multirow[t]{2}{*}{2.5} & Bias & -0.1621 & 0.0052 & -.00006 & -0.0879 & 0.0010 & 0.0071 \\
\hline & & RMSEs & 1.1603 & 1.1058 & 1.2293 & 0.8236 & 0.7823 & 0.8742 \\
\hline & \multirow[t]{2}{*}{3.5} & Bias & -0.1635 & -0.0045 & -0.0097 & -0.0826 & -0.0043 & -0.0060 \\
\hline & & RMSEs & 1.0567 & 1.0512 & 1.2127 & 0.7544 & 0.7424 & 0.8457 \\
\hline & \multirow[t]{2}{*}{5} & Bias & -0.1418 & 0.0104 & 0.01018 & -0.0852 & -0.0090 & -0.0071 \\
\hline & & RMSEs & 1.0214 & 1.0333 & 1.2134 & 0.7252 & 0.7291 & 0.8485 \\
\hline \multicolumn{3}{|c|}{ Parameters } & \multicolumn{3}{|c|}{$\mathrm{n}=500$} & \multicolumn{3}{|c|}{$\mathrm{n}=1000$} \\
\hline $\mathrm{a}$ & $\mathrm{q}$ & & MME & LME & TLME & MME & LME & TLME \\
\hline \multirow[t]{6}{*}{2.5} & \multirow[t]{2}{*}{2.5} & Bias & -0.0310 & -0.0004 & 0.0015 & -0.0154 & -0.0011 & -0.0019 \\
\hline & & RMSEs & 0.6482 & 0.4411 & 0.4260 & 0.4611 & 0.3135 & 0.3018 \\
\hline & \multirow[t]{2}{*}{3.5} & Bias & -0.0247 & -0.0026 & -0.0004 & -0.0068 & 0.0037 & 0.0056 \\
\hline & & RMSEs & 0.4792 & 0.3910 & 0.4080 & 0.3467 & 0.2768 & 0.2851 \\
\hline & \multirow[t]{2}{*}{5} & Bias & -0.0152 & 0.0021 & 0.00211 & -0.0138 & -0.0043 & -0.0041 \\
\hline & & RMSEs & 0.3983 & 0.3538 & 0.3866 & 0.2834 & 0.2529 & 0.2767 \\
\hline \multirow[t]{6}{*}{3.5} & \multirow[t]{2}{*}{2.5} & Bias & -0.0236 & -0.0027 & -0.0035 & -0.0108 & -0.0007 & 0.0003 \\
\hline & & RMSEs & 0.4556 & 0.3812 & 0.4035 & 0.3263 & 0.2682 & 0.2798 \\
\hline & \multirow[t]{2}{*}{3.5} & Bias & -0.0163 & 0.0006 & -0.0004 & -0.0124 & -0.0047 & -0.0062 \\
\hline & & RMSEs & 0.3777 & 0.3469 & 0.3830 & 0.2678 & 0.2459 & 0.2709 \\
\hline & 5 & Bias & -0.0159 & 0.0001 & 0.0002 & -0.0093 & -0.0021 & -0.0042 \\
\hline & & RMSEs & 0.3378 & 0.3290 & 0.3780 & 0.2397 & 0.2316 & 0.2638 \\
\hline 5 & 2.5 & Bias & -0.0083 & -0.0011 & -0.0017 & -0.0087 & -0.0004 & -0.0018 \\
\hline & & RMSEs & 0.2654 & 0.2470 & 0.2733 & 0.2669 & 0.2483 & 0.2718 \\
\hline & 3.5 & Bias & -0.0165 & -0.0005 & -0.0008 & -0.0094 & -0.0021 & -0.0037 \\
\hline & & RMSEs & 0.3389 & 0.3332 & 0.3789 & 0.2414 & 0.2364 & 0.2671 \\
\hline & 5 & Bias & -0.0060 & 0.0017 & 0.0027 & -0.0070 & .00005 & -0.0008 \\
\hline & & RMSEs & 0.2279 & 0.2294 & 0.2668 & 0.2280 & 0.2283 & 0.2616 \\
\hline
\end{tabular}


Table A.5: Mean, S.D, CV, CS and CK different parametric values assuming MMEs, LMEs and TLMEs

\begin{tabular}{|c|c|c|c|c|c|c|c|}
\hline \multirow[t]{2}{*}{$\mathrm{a}$} & \multirow[t]{2}{*}{$\mathrm{b}$} & \multirow[t]{2}{*}{$\mathrm{q}$} & Mean & S.D & $\mathrm{CV}$ & $\mathrm{CS}$ & CK \\
\hline & & & \multicolumn{5}{|c|}{ Method of Moment Estimates } \\
\hline 2.5 & 2.5 & 2.5 & 1.74618 & 0.96423 & 0.55219 & 1.59264 & 9.96322 \\
\hline 3.5 & & & 1.87901 & 0.73405 & 0.39066 & 0.87000 & 5.18644 \\
\hline 5.0 & & & 2.01460 & 0.55391 & 0.27495 & 0.41096 & 3.83527 \\
\hline 10 & & & 2.22241 & 0.31206 & 0.14041 & -0.11015 & 3.47769 \\
\hline \multirow[t]{4}{*}{2.5} & 2.5 & 2.5 & 1.74618 & 0.96423 & 0.55219 & 1.59264 & 9.96322 \\
\hline & 3.5 & & 2.44465 & 1.34992 & 0.55219 & 1.59264 & 9.96322 \\
\hline & 5.0 & & 3.49236 & 1.92847 & 0.55219 & 1.59264 & 9.96322 \\
\hline & 10 & & 6.98473 & 3.85693 & 0.55219 & 1.59264 & 9.96322 \\
\hline \multirow[t]{4}{*}{2.5} & 2.5 & 2.5 & 1.74618 & 0.96423 & 0.55219 & 1.59264 & 9.96322 \\
\hline & & 3.5 & 1.46679 & 0.74442 & 0.50751 & 1.08950 & 5.67080 \\
\hline & & 5.0 & 1.23674 & 0.59315 & 0.47961 & 0.81551 & 4.25391 \\
\hline & & 10 & 0.90892 & 0.41060 & 0.45174 & 0.56301 & 3.35399 \\
\hline a & $\mathrm{b}$ & $q$ & \multicolumn{5}{|c|}{ L-Moment Estimates } \\
\hline 2.5 & 2.5 & 2.5 & 1.74618 & 0.50944 & 0.29174 & 0.18854 & 0.16014 \\
\hline 3.5 & & & 1.87901 & 0.40268 & 0.21430 & 0.11113 & 0.14526 \\
\hline 5.0 & & & 2.01460 & 0.30854 & 0.15315 & 0.05080 & 0.14071 \\
\hline 10 & & & 2.22241 & 0.17429 & 0.07842 & -0.02203 & 0.14334 \\
\hline \multirow[t]{4}{*}{2.5} & 2.5 & 2.5 & 1.74618 & 0.50944 & 0.29174 & 0.18854 & 0.16014 \\
\hline & 3.5 & & 2.44465 & 0.71321 & 0.29174 & 0.18854 & 0.16014 \\
\hline & 5.0 & & 3.49236 & 1.01888 & 0.29174 & 0.18854 & 0.16014 \\
\hline & 10 & & 6.98473 & 2.03776 & 0.29174 & 0.18854 & 0.16014 \\
\hline \multirow[t]{4}{*}{2.5} & 2.5 & 2.5 & 1.74618 & 0.50944 & 0.29174 & 0.18854 & 0.16014 \\
\hline & & 3.5 & 1.46679 & 0.40484 & 0.27600 & 0.15103 & 0.14100 \\
\hline & & 5.0 & 1.23674 & 0.32781 & 0.26506 & 0.12369 & 0.12821 \\
\hline & & 10 & 0.90892 & 0.23011 & 0.25316 & 0.09272 & 0.11494 \\
\hline $\mathrm{a}$ & $\mathrm{b}$ & $q$ & \multicolumn{5}{|c|}{ TL-Moment Estimates } \\
\hline 2.5 & 2.5 & 2.5 & 1.65012 & 0.25671 & 0.15557 & 0.10855 & 0.07708 \\
\hline 3.5 & & & 1.83426 & 0.20651 & 0.11258 & 0.06144 & 0.07183 \\
\hline 5.0 & & & 1.99892 & 0.15907 & 0.07958 & 0.02539 & 0.07064 \\
\hline 10 & & & 2.22625 & 0.08958 & 0.04024 & -0.01743 & 0.07239 \\
\hline \multirow[t]{4}{*}{2.5} & 2.5 & 2.5 & 1.65012 & 0.25671 & 0.15557 & 0.10855 & 0.07708 \\
\hline & 3.5 & & 2.31018 & 0.35939 & 0.15557 & 0.10855 & 0.07708 \\
\hline & 5.0 & & 3.30025 & 0.51342 & 0.15557 & 0.10855 & 0.07708 \\
\hline & 10 & & 6.60051 & 1.02685 & 0.15557 & 0.10855 & 0.07708 \\
\hline \multirow{4}{*}{2.5} & 2.5 & 2.5 & 1.65012 & 0.25671 & 0.15557 & 0.10855 & 0.07708 \\
\hline & & 3.5 & 1.40564 & 0.20865 & 0.14844 & 0.08752 & 0.06943 \\
\hline & & 5.0 & 1.19619 & 0.17147 & 0.14334 & 0.07193 & 0.06422 \\
\hline & & 10 & 0.88759 & 0.12219 & 0.13767 & 0.05396 & 0.05870 \\
\hline
\end{tabular}

